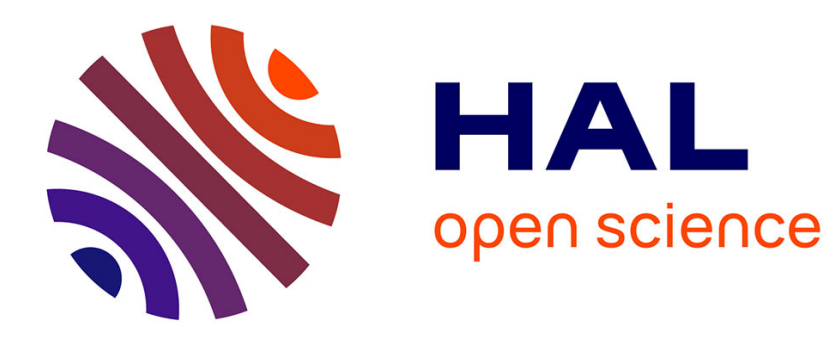

\title{
Jean-Loup BOURGET, Rebecca
}

Katalin Pór

\section{To cite this version:}

Katalin Pór. Jean-Loup BOURGET, Rebecca. 1895 revue d'histoire du cinéma, 2018, 85, pp.205-207. 10.4000/1895.6675 . hal-03213437

\section{HAL Id: hal-03213437 \\ https://hal.science/hal-03213437}

Submitted on 4 May 2021

HAL is a multi-disciplinary open access archive for the deposit and dissemination of scientific research documents, whether they are published or not. The documents may come from teaching and research institutions in France or abroad, or from public or private research centers.
L'archive ouverte pluridisciplinaire HAL, est destinée au dépôt et à la diffusion de documents scientifiques de niveau recherche, publiés ou non, émanant des établissements d'enseignement et de recherche français ou étrangers, des laboratoires publics ou privés. 


\section{JEAN-LOUP BOURGET, REBECCA}

\section{Katalin Por}

AFRHC | «1895»

2018/2 n 85 | pages 205 à 207

ISSN 0769-0959

Article disponible en ligne à l'adresse :

https://www.cairn.info/revue-1895-2018-2-page-205.htm

Distribution électronique Cairn.info pour AFRHC.

(C) AFRHC. Tous droits réservés pour tous pays.

La reproduction ou représentation de cet article, notamment par photocopie, n'est autorisée que dans les limites des conditions générales d'utilisation du site ou, le cas échéant, des conditions générales de la licence souscrite par votre établissement. Toute autre reproduction ou représentation, en tout ou partie, sous quelque forme et de quelque manière que ce soit, est interdite sauf accord préalable et écrit de l'éditeur, en dehors des cas prévus par la législation en vigueur en France. Il est précisé que son stockage dans une base de données est également interdit. 


\section{Mille huit cent quatre-vingt-quinze}

Revue de l'association française de recherche sur

I'histoire du cinéma

$85 \mid 2018$

Varia

\section{Jean-Loup Bourget, Rebecca}

\section{Katalin Pór}

Édition électronique
URL : http://journals.openedition.org/1895/6675

ISSN : 1960-6176

Éditeur

Association française de recherche sur l'histoire du cinéma (AFRHC)

Édition imprimée

Date de publication : 30 septembre 2018

Pagination : 205-207

ISBN : 978-2-37029-085-4

ISSN : 0769-0959

Référence électronique

Katalin Pór, "Jean-Loup Bourget, Rebecca », 1895. Mille huit cent quatre-vingt-quinze [En ligne],

85 | 2018, mis en ligne le 30 septembre 2018, consulté le 21 février 2019. URL : http://

journals.openedition.org/1895/6675

Ce document a été généré automatiquement le 21 février 2019.

(c) $\mathrm{AFRHC}$ 


\title{
Jean-Loup Bourget, Rebecca
}

\author{
Katalin Pór
}

\section{RÉFÉRENCE}

Jean-Loup Bourget, Rebecca, Paris, Vendémiaire, « Contrechamp », 2017, 142 p.

1 Que peut-on encore dire sur l'œuvre d'Hitchcock en général, et sur Rebecca en particulier? Des exégèses formalistes aux analyses genrées, en passant par les nombreux ouvrages d'entretiens - du cinéaste ou de ses collaborateurs -, la bibliographie sur le cinéaste est imposante, au point d'ailleurs que Jean-Loup Bourget ne cherche pas à en faire la présentation exhaustive, n'indiquant en bibliographie que les ouvrages qu'il mobilise directement pour son analyse. Pour répondre à ce défi, Bourget, un des grands spécialistes français du cinéma américain, repart dans cet ouvrage de la question historiquement au fondement de l'intérêt français pour le cinéaste, à savoir celle de l'auteur. Il propose de l'appréhender à travers l'analyse d'un seul film, Rebecca, communément admis comme "l'œuvre pivot » du cinéaste (premier film hollywoodien du cinéaste, il porterait encore les marques de la période britannique, tout en annonçant les transformations hollywoodiennes de son œuvre), sur lequel Bourget parvient à renouveler le regard, grâce à un texte relativement bref mais foisonnant, fourmillant de pistes visant à restituer les différentes lignées et réseaux dans lesquels elle s'inscrit. L'ouvrage se divise en quatre parties - «Genèses ", «Lectures », « La première personne du singulier » et « Romans familiaux »-, dissymétriques tant par leur taille que par leur principe d'organisation. Si les deux premières se structurent autour de méthodes d'analyse - analyse génétique pour la première, analyse textuelle pour la seconde -, la troisième se penche plus particulièrement sur les procédés narratifs, tandis que la dernière conclut l'ouvrage sur l'inscription du film dans un nouveau réseau d'œuvres lié aux romans des sœurs Brontë. Bourget prend ainsi le parti de ne pas faire avancer son propos et ses analyses de manière linéaire, ni même systématique, mais plutôt à travers une série d'élargissements, de décadrages et de retours. 
2 Ainsi, le premier élargissement qu'il mène est à la fois rétrospectif et latéral. La première partie de l'ouvrage, "Genèses ", se penche en effet sur le processus d'élaboration de l'œuvre. Pour ce faire, elle restitue aussi bien le contexte de production (l'arrivée d'Hitchcock à Hollywood, la genèse du projet d'adaptation du roman de du Maurier, la stratégie de production de Selznick, etc.) que les phénomènes d'interférences avec les productions médiatiques et spectaculaires qui lui sont contemporaines. Soulignant la part des différentes instances impliquées dans la création (compositeur, scénaristes, etc.), ainsi que le rôle joué par l'adaptation radiophonique produite, réalisée et interprétée peu de temps avant par Orson Welles, Bourget y affirme néanmoins que le film doit avant tout être mis au crédit des trois instances auctoriales que sont le réalisateur Alfred Hitchcock, le producteur David O. Selznick, et la romancière Daphné du Maurier. Cette thèse s'appuie sur une analyse de divers documents de production (mémos de Selznick, versions de scénario, correspondance avec la PCA - Production Code Administration -, matériau publicitaire, etc.). Ces documents lui permettent en effet de mettre à jour les divergences de conception entre Hitchcock et Selznick et les modifications scénaristiques qui en découlent, les discussions avec le Hays office et leurs conséquences sur le récit notamment autour du suicide/meurtre de Rebecca -, ou encore les hésitations autour du casting; ils donnent également à voir les instances créatives valorisées au moment de la sortie du film. Ainsi, si ces documents offrent des éclairages sur la part créative qu'on peut allouer à chacun, ils donnent surtout à voir les processus constants de négociations et de rapports de force à travers lesquels le film s'élabore. On regrette que leur usage ne soit pas plus important, tant il apparaît une fois de plus qu'ils constituent des outils éminemment précieux pour qui souhaite renouveler le regard sur des œuvres aussi connues.

3 Le propos avance ensuite par une série de décadrages. Bourget opère en effet sa démonstration à travers l'inscription $\mathrm{du}$ film dans divers réseaux: thématiques, iconographiques, stylistiques ou génériques. Celui du gothique, reliant le film, via le motif du portrait peint, aux œuvres d'Edgar Poe et Oscar Wilde ; celui du stream of consciousness, qui court des romans de Virginia Woolf et James Joyce jusqu'à des films tels que Murder (Hitchcock), Strange Interlude (Robert Z. Leonard), ou encore The Power and the Glory (William K. Howard) ; celui du motif du feu purificateur, reliant le film au Frankenstein de James Whale, voire à la tradition picturale "ruiniste »; ou encore celui des œuvres des sœurs Brontë, intégrant aussi bien Jane Eyre et Wuthering Heights (les Hauts de Hurlevent) que leurs adaptations cinématographiques - notamment celle réalisée par William Wyler - ainsi que Devotion, biopic Warner sur les sœurs Brontë réalisé par Curtis Bernhardt. Bourget s'emploie également, de manière plus attendue, à relier Rebecca aux autres réalisations d'Hitchcock: à Vertigo pour le motif du modelage de la jeune femme, à Suspicion pour la veine du cinéma subjectif, ou encore à Easy Virtue et The Skin Game pour la satire sociopolitique. Ce même mouvement de décentrage du propos commande également l'appréhension de l'œuvre même : fidèle à la démarche qu'il promeut depuis la Norme et la marge (Nathan, 1998), Bourget accorde une attention particulière aux éléments périphériques de l'œuvre : le titre, le générique, ainsi que les séquences d'ouverture et de clôture font ainsi l'objet d'analyses détaillées.

4 Enfin, le troisième mouvement de décentrement consiste à faire avancer la démonstration à travers une analyse critique des lectures précédentes. Dans la partie «Lectures", Bourget revient en détail sur deux modèles d'analyse de l'œuvre du cinéaste : la lecture théologico-formaliste portée par les «Hitchcocko-Hawksiens » des 
Cahiers du Cinéma - notamment Rohmer, Truffaut et Chabrol -, et la lecture féministe, à travers la discussion de l'ouvrage de Tania Modleski, The Women Who Knew Too Much. Hitchcock and Feminist Theory. Il ne s'agit pas pour lui de retracer l'histoire de l'exégèse du film, mais plutôt de trouver dans ces lectures des éléments permettant de faire progresser la réflexion sur la question de départ - celle de l'auteur. Celle-ci se trouve ensuite progressivement décalée et remplacée par celle de la place du film dans l'œuvre du cinéaste, et plus particulièrement son rapport aux contextes britannique et américain, permettant in fine à Bourget de proposer une interprétation valorisant le discours sociopolitique du film, et la proximité avec les gravures et tableaux satiriques de Hogarth. Jean-Loup Bourget défend, depuis au moins 1988 et la sortie du Classical Hollywood Cinema. Film Style and Mode of Production to 1960 de Bordwell, Staiger et Thompson, la pertinence et la nécessité d'une histoire du cinéma centrée sur l'analyse textuelle des œuvres. L'ouvrage s'inscrit fermement dans ce parti-pris méthodologique: l'ouverture est consacrée à l'analyse du plan illustrant la couverture, représentant « Je » et Mrs Danvers ; de même, les analyses de séquences minutieuses abondent. Ce travail d'analyse filmique se trouve cependant systématiquement enrichi par le mouvement permanent d'élargissement de la focale. L'ouvrage a ainsi pour mérite essentiel de se présenter comme un texte ouvert à de multiples pistes, bien plus que comme une interprétation fermée. Il fait une fois de plus la démonstration de la pertinence de la double démarche consistant à retracer la genèse de l'œuvre, ainsi qu'à restituer le contexte intermédial dans lequel elle s'inscrit, comme modalité d'enrichissement et de renouvellement de l'analyse filmique. 